\title{
NMR Measurement of the Magnetic Field Correlation Function in Porous Media
}

\author{
H. Cho* and Yi-Qiao Song \\ Schlumberger-Doll Research, One Hampshire Street, Cambridge, Massachusetts 02139, USA
}

(Received 15 September 2007; published 15 January 2008)

\begin{abstract}
The structure factor provides a fundamental characterization of porous and granular materials as it is the key for solid crystals via measurements of $x$-ray and neutron scattering. Here, we demonstrate that the structure factor of the granular and porous media can be approximated by the pair correlation function of the inhomogeneous internal magnetic field, which arises from the susceptibility difference between the pore filling liquid and the solid matrix. In-depth understanding of the internal field is likely to contribute to further development of techniques to study porous and granular media.
\end{abstract}

DOI: 10.1103/PhysRevLett.100.025501

Sand piles, rocks, and colloids share a common structure element that they are examples of materials formed by aggregation of granular particles in another medium. Their physical properties are critically dependent on the grain-grain interactions and the structure of the grain arrangement [1-3]. Details of the packing are important for the presence of heterogeneous force causing jamming [4,5], unique acoustic properties [6], and clustering [1]. It was found that the spatial correlation functions of the electromagnetic fields in such porous media can be useful to predict fluid flow and structure factor. For example, twodimensional images have been used to construct the spatial correlation functions of porous media and to infer material properties [7-10], and it was shown that correlation length of electric field is similar to the hydraulic radius [11]. In biomedical imaging, magnetic field correlation was examined for a potential quantitative assessment of iron in brain [12]. It was shown that the magnetic field correlation function is closely related to the structure factor of the pore space $[13,14]$. Thus it is desirable to devise an experimental method to directly measure the correlation function of the electromagnetic field in such media.

In this Letter, we present a NMR method to measure the spatial magnetic correlation function in model porous materials. When a granular sample is placed in a uniform magnetic field, the solid grain and the interstitial material are magnetized differently due to a susceptibility contrast, producing an inhomogeneous magnetic field in the pore space - often called internal field. We use nuclear spins to probe the magnetic field difference between two positions by the following method. First, we employ pulsed field gradient (PFG) NMR to select spins by their translational diffusion displacement. For this experiment, the effect of the internal field is nullified. Then, we perform a similar experiment but with the internal field effect. The field difference between the two positions causes a signal decay that is related to the magnetic field correlation at that displacement. We show that the method is robust for different grain sizes, diffusion, encoding times, and displacement resolution and reliably obtains surface-to-volume ratio (SVR).
PACS numbers: 76.60.Jx, 61.05.Qr, 61.43.Gt, 75.75.+a

Conventional translation diffusion measurements often use stimulated echo sequence,

$$
\pi / 2-t-\pi / 2-\Delta-\pi / 2-t-\text { echo, }
$$

where $\pi / 2$ denotes a rf pulse that rotates spin vector by $90^{\circ}$. During the two time periods $t$, two identical field gradient pulses were applied to modulate the spin magnetization. Typically, $\Delta$ is the dominant diffusion time much longer than $t$. A spin moving a displacement of $\mathbf{r}$ accumulates a net phase of $\mathbf{q} \cdot \mathbf{r}$ where $\mathbf{q}=\gamma \delta \mathbf{g}(\gamma, \mathbf{g}$, and $\delta$ are the gyromagnetic ratio, the strength, and the duration of applied field gradient pulse, respectively). The resulting echo signal for the ensemble of spins is

$$
E(\mathbf{q}, \Delta)=\int P(\mathbf{r}, \Delta) e^{i \mathbf{q} \cdot \mathbf{r}} d \mathbf{r},
$$

$P(\mathbf{r}, \Delta)$ is the average propagator, the conditional probability for a molecule diffusing a displacement of $\mathbf{r}$ during the time $\Delta$ [15]. A Fourier transform of $E(\mathbf{q}, \Delta)$ will produce $P(\mathbf{r}, \Delta)$ which is proportional to the number of spins that have traversed a distance of $\mathbf{r}$ during $\Delta$.

To consider that the internal field $B^{i}$ is effective during the $t$ periods, signal will be further weighted by the internal field effects as the spins diffuse from position $\mathbf{R}$ to $\mathbf{R}+\mathbf{r}$,

$$
E^{\prime}(\mathbf{q}, \Delta)=\int P e^{i \mathbf{q} \cdot \mathbf{r}} e^{i \gamma t\left(B_{z}^{i}(\mathbf{r}+\mathbf{R})-B_{z}^{i}(\mathbf{R})\right)} d \mathbf{r} d \mathbf{R} .
$$

Fourier transform of $E^{\prime}$ will determine $P^{\prime}$, similar to $P$ with a small internal magnetic field perturbation: $P^{\prime}=$ $P(\mathbf{r}, \Delta) \int \exp \left[i \gamma t\left(B_{z}^{i}(\mathbf{r}+\mathbf{R})-B_{z}^{i}(\mathbf{R})\right)\right] d \mathbf{R}$, where $B_{z}^{i}$ is the internal field component along the applied field direction. The ratio of the two measurements gives

$$
P^{\prime} / P=\int e^{i \gamma t\left(B_{z}^{i}(\mathbf{r}+\mathbf{R})-B_{z}^{i}(\mathbf{R})\right)} d \mathbf{R} \equiv C(\mathbf{r}) .
$$

When the exponent is small, Eq. (4) can be Taylor expanded to second order,

$$
C(\mathbf{r}) \approx 1-\gamma^{2} t^{2}\left\langle B_{z}^{i 2}\right\rangle+\gamma^{2} t^{2}\left\langle B_{z}^{i}(\mathbf{r}) B_{z}^{i}(0)\right\rangle,
$$

where $\left\langle B_{z}^{i}(\mathbf{r}) B_{z}^{i}(0)\right\rangle$ is the pair correlation function of $B_{z}^{i}$ 
and $\left\langle B_{z}^{i 2}\right\rangle$ is the second moment. Since the experiments are performed with fixed $\Delta$, the measured $P$ and $P^{\prime}$ are not affected by surface relaxation.

Samples were prepared with randomly packed glass beads (Duke Scientific Corp.) of nominal radius $\left(r_{0}\right)$ of 25 and $50 \mu \mathrm{m}$ and saturated with water in $0.5 \mathrm{~cm}$ glass tubes. Experiments were performed at a 2-T horizontal bore magnet (Nalorac Cryogenics) operating at a proton frequency of $85.1 \mathrm{MHz}$. Spectral linewidth for the 25 and $50 \mu \mathrm{m}$ beads is 670 and $290 \mathrm{~Hz}$, and the nominal $T_{1} 0.5$ and $0.8 \mathrm{~s}$, respectively. A Bruker Biospec spectrometer and a homebuilt rf probe with a $1 \mathrm{~cm}$-diameter saddle coil were used. The sensitive region of the coil was $\sim 2 \mathrm{~cm}$, smaller than the length of sample $4.5 \mathrm{~cm}$ to avoid sample boundary effect.

Figure 1 shows the experimental pulse sequences for measuring $P$ and $P^{\prime}$, respectively. Sequence 1(a) introduces a $\pi$ pulse in the middle of the first two $\pi / 2$ pulses $(\tau=$ $\left.\tau^{\prime}\right)$ to refocus the magnetization in order to cancel the phase due to $B_{z}^{i}$. We call this the balanced sequence and used it to measure $P$. Sequence 1(b) is imbalanced, $\tau^{\prime} \neq \tau$, so that the net phase due to $B_{z}^{i}$ is proportional to $t=\tau^{\prime}-$ $\tau$. This sequence allows an easy adjustment of $t$ while keeping identical the diffusion weightings due to PFG.
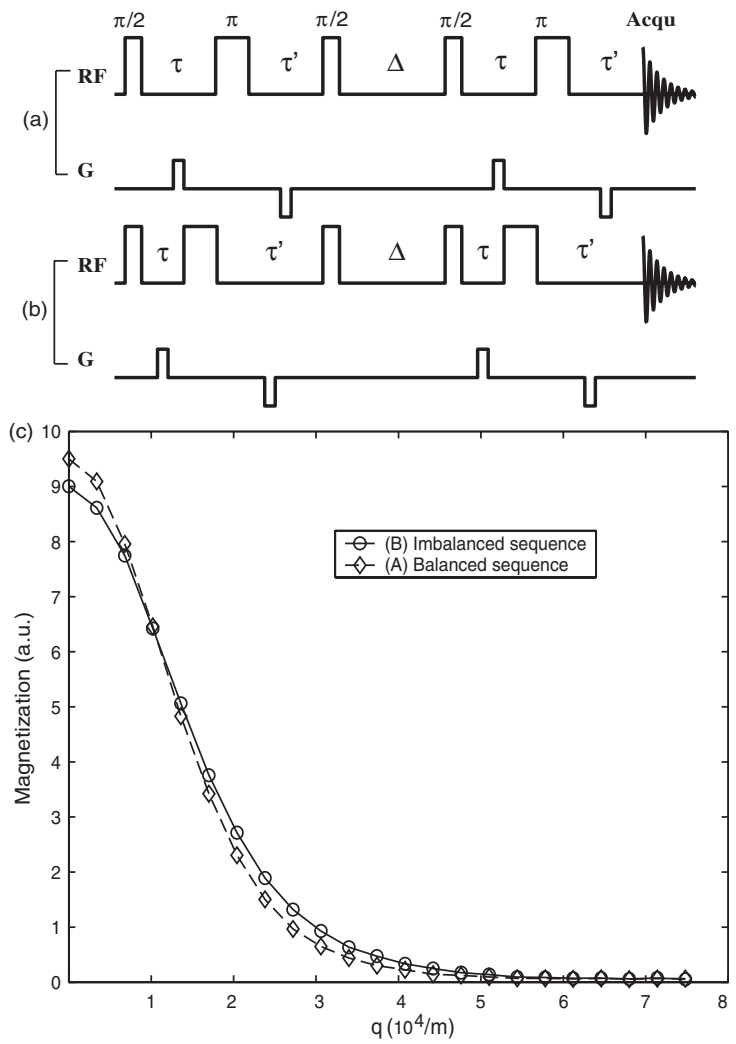

FIG. 1. Top figures show pulse sequences to measure pair correlation function of internal magnetic field: (a) balanced sequence and (b) imbalanced sequence. (c) Typical experimental signals with the corresponding pulse sequences. Encoding time $t=300 \mu \mathrm{s}$ and $\Delta=70 \mathrm{~ms}$ and gradient along $z$.
Gradient pulses of length 2 or $1.5 \mathrm{~ms}$ are applied up to $92 \mathrm{G} / \mathrm{cm}$ to achieve a displacement resolution of 6.3 or $8.5 \mu \mathrm{m}$, respectively.

For isotropic materials, it is adequate to use gradient along one direction in each experiment. Since the propagator is a product of the propagator along each direction, $P(x, y, z)=P(x) P(y) P(z)$ where $x, y$, and $z$ are displacement along the three coordinates. In this case, $P$ and $P^{\prime}$ are measured only as a function of the displacement along the gradient direction $\left(r_{\|}\right)$; thus, we define $C 1\left(r_{\|}\right)$:

$$
\begin{aligned}
C 1\left(r_{\|}\right) & \equiv P^{\prime}\left(r_{\|}\right) / P\left(r_{\|}\right) \\
& \approx b+\gamma^{2} t^{2}\left\langle\int P\left(\mathbf{r}_{\perp}, \Delta\right) B_{z}^{i}(\mathbf{r}) B_{z}^{i}(0) d \mathbf{r}_{\perp}\right\rangle,
\end{aligned}
$$

where $b \approx 1-\gamma^{2} t^{2}\left\langle\left(B_{z}^{i}\right)^{2}\right\rangle$ is independent of $r_{\|}$, and the bracket refers to an ensemble average. The subscripts " $\perp$ " and " $\|$ " denote the components perpendicular and parallel to the applied gradient, respectively. Since $\mathbf{r}$ has two components $\left(r_{\|}\right.$and $\mathbf{r}_{\perp}$ ), the integration of $\mathbf{r}_{\perp}$ means that $C 1$ is only a function of $r_{\|}$. For those spins with a specific $r_{\|}$, the transverse displacement may cause additional signal decay reflected in the $\mathbf{r}_{\perp}$ integral of Eq. (7).

Typical echo signals are shown in Fig. 1. The reduction of signal at $q=0$ for the imbalanced sequence is indicative of the effect of internal field and consistent with previous measurements $[16,17]$. We also observed a slower decay of the imbalanced sequence in $q$ space than that of the balanced one and the crossover of the two signals which can be understood as follows. For nonmoving spins

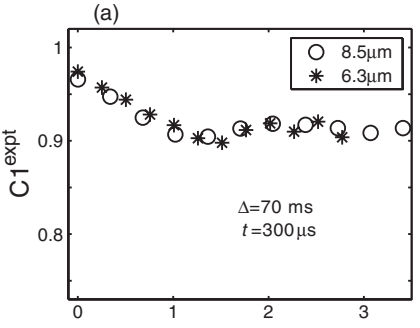

(c)

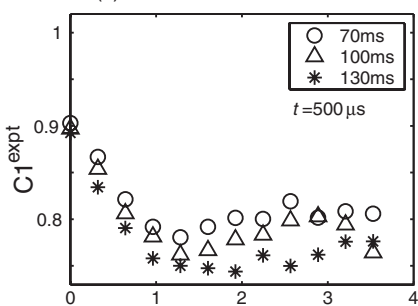

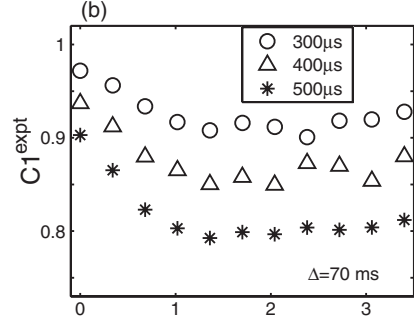

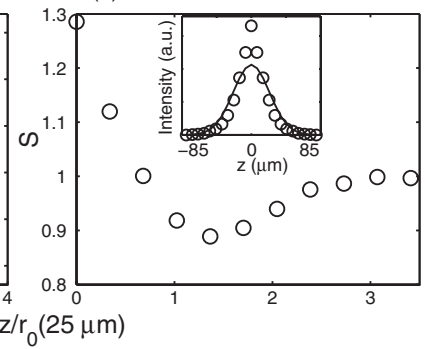

FIG. 2. Measurements of $C 1\left(r_{\|}\right)$with various experimental parameters for the $25 \mu \mathrm{m}$ beads sample (a)-(c). (a) Resolution, (b) encoding time $t$, (c) diffusion time $(\Delta)$. (d) The structure factor from measured propagator using Eq. (8). Inset of (d): The measured propagator for the $25 \mu \mathrm{m}$ beads sample (circles) with $t=0 \mathrm{~s}, \Delta=130 \mathrm{~ms}$, and $8.3 \mu \mathrm{m}$ resolution and the Gaussian propagator (line) at the corresponding diffusion time. 
$(\mathbf{r}=0), P$ and $P^{\prime}$ are identical because there is no net phase in either case. This equality of $P(0)$ and $P^{\prime}(0)$ requires the areas under the two data sets in $q$ domain to be the same due to the Fourier relationships [Eqs. (2) and (3)]. Thus, the imbalanced data must decay slower and crosses over the balanced data.

Next, the echo signals from both sequences are Fourier transformed to derive $P^{\prime}$ and $P$, then $C 1\left(r_{\|}\right)$, shown in Fig. 2. Initial linear decay followed by a minimum at $z \sim$ $1.5 r_{0}$ is observed for every experiment. Figure 2(a) shows a consistent $C 1(z)$ at different resolution with other parameters fixed. Figure 2(b) shows $C 1(z)$ as a function of $t$. It is observed that the values of $C 1(0)$ are not exactly one and decreases as $t$ increases. Higher resolution data show a slightly larger $C 1(0)$ value in 2(a) suggesting this is partly due to the averaging effect of the finite resolution. Also, diffusion transverse to the direction of the gradients also causes signal decay. This is consistent with the decreasing $C 1(0)$ values with larger $t$ shown in 2(b). Figure 2(c) shows the $\Delta$ dependence of $C 1(z)$ with a slightly decreasing baseline value at larger $\Delta$ also due to transverse diffusion.

The inset of Fig. 2(d) shows measured average propagator (" $\bigcirc$ ") for the $25 \mu \mathrm{m}$ beads sample at $\Delta=130 \mathrm{~ms}$ superimposed with the corresponding free diffusion Gaussian propagator. The narrower and sharper peak of $P$ near the origin indicates the effect of restricted diffusion. It has been recognized that the averaged diffusion propagator in porous media can be modeled as a Gaussian modulated by the presence of excluded regions $[9,18-$ 20]; i.e.,

$$
P(\mathbf{r}, \Delta) \propto S(\mathbf{r}) \exp \left[-\mathbf{r}^{2} / 4 D(\Delta) \Delta\right]
$$

where $D(\Delta)$ is diffusion coefficient at the diffusion time $\Delta$ and $S(\mathbf{r})$ is the connected pore-space structure factor. We divided the measured $P$ for the $25 \mu \mathrm{m}$ beads sample by the Gaussian propagator corresponding to the diffusion time $\Delta$ to obtain $S\left(r_{\|}\right)$from Eq. (8), which are shown in Fig. 2(d). A qualitative resemblance is observed with known structure factor for random bead pack with an initial linear decay followed by a minimum at $r_{\|} \sim 1.5 r_{0}$ and also with the internal field correlation [Figs. 2(a)-2(c)].

To extract the internal field correlation function $K\left(r_{\|}\right)$ from $C 1\left(r_{\|}\right)$, we estimated the baseline $(b)$ value of $C 1\left(r_{\|}\right)$ at large $r_{\|}$to obtain $K\left(r_{\|}\right)=\left[C 1\left(r_{\|}\right)-b\right] /[C 1(0)-b]$, removing the effects of finite resolution and diffusion during the encoding period, and the results are shown in Fig. 3. $K\left(r_{\|}\right)$in Figs. 3(a)-3(c) are observed to reveal a scaling behavior after the renormalization, independent of experimental parameters. Figure 3(d) shows $K\left(r_{\|}\right)$measurement for samples of different radius beads to demonstrate its sensitivity to pore sizes.

To compare with experimental results, we numerically evaluated the internal field on the dense random packing model of hard spheres [21] by a superposition of fields from dipoles located at the centers of the spheres with a

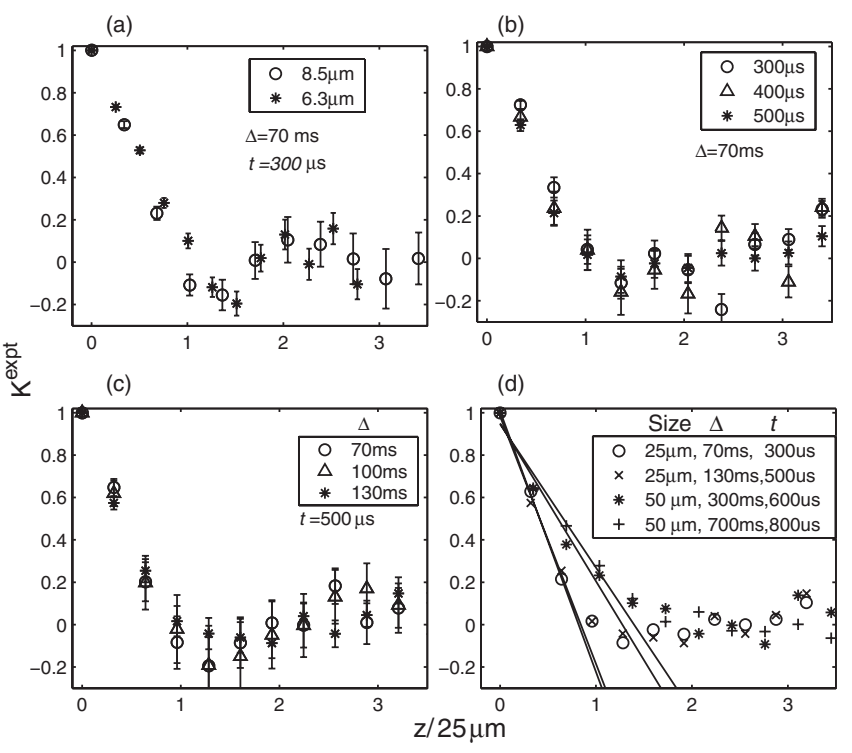

FIG. 3. A scaling behavior of $K\left(r_{\|}\right)$for various experimental parameters performed with the $25 \mu \mathrm{m}$ beads sample (a)-(c). (a) Resolution, (b) encoding time $t$, (c) diffusion time $(\Delta)$. (d) Comparison between the two samples with bead radius 25 and $50 \mu \mathrm{m}$ and linear fits for initial decay regime to extract SVR.

dipole moment $\mathbf{m}=4 \pi / 3 r_{0}^{3} \Delta \chi \mathbf{B}_{0}$, where the susceptibility difference of the grain and medium is $\Delta \chi$, and $\Delta \chi \ll 1$. To calculate the field correlation function, we randomly sampled 100000 points in the sphere pack and evaluated the field at each point [14]. For every pair of points $\left(\mathbf{R}_{i}\right.$ and $\left.\mathbf{R}_{j}\right), B_{z}^{i}\left(\mathbf{R}_{i}\right) B_{z}^{i}\left(\mathbf{R}_{j}\right) P\left(\mathbf{r}_{\perp}\right)$ was evaluated and binned by the distance of the two points along the gradient direction, $\mathcal{K}=\sum_{i j} B_{z}^{i}\left(\mathbf{R}_{i}\right) B_{z}^{i}\left(\mathbf{R}_{j}\right) P\left(\mathbf{r}_{\perp}\right) / n^{\prime}$ where $n^{\prime}$ is the number of pairs with distance $r_{\|}$, and $\mathbf{r}_{\perp}$ is the component of $\mathbf{R}_{i}-$ $\mathbf{R}_{j}$ perpendicular to the gradient. Then,

$$
K^{\text {calc }}\left(r_{\|}\right)=\mathcal{K}\left(r_{\|}\right) / \mathcal{K}(0) .
$$

Figure 4 shows the comparison of the numerical calculations and the experimental results. Figure 4(a) shows numerical internal field correlations $\left(r_{\|}=z\right.$, along the direction of the static magnetic field) as a function of porosity of the random packing. The porosity of the bead pack was varied by randomly removing beads in the pack. Decreasing initial slope is observed as the porosity increases which also reduces SVR [22]. Figure 4(b) shows a linear relationship between $K^{\prime}$ (the initial slope of $K$ ) and SVR, i.e.,

$$
K^{\prime} \approx A / 4 \mathrm{SVR},
$$

and the slope $A$ was found $1.5,1.1$, and 1.1 along $z, x$, and $y$, respectively.

Figure 4(c) shows the experimentally measured $C$ along different gradient directions. The reduction $[C 1(0)-$ $C 1(\infty)=\mathcal{K}(0)]$ is the strongest along $z$ and the anisotropy 

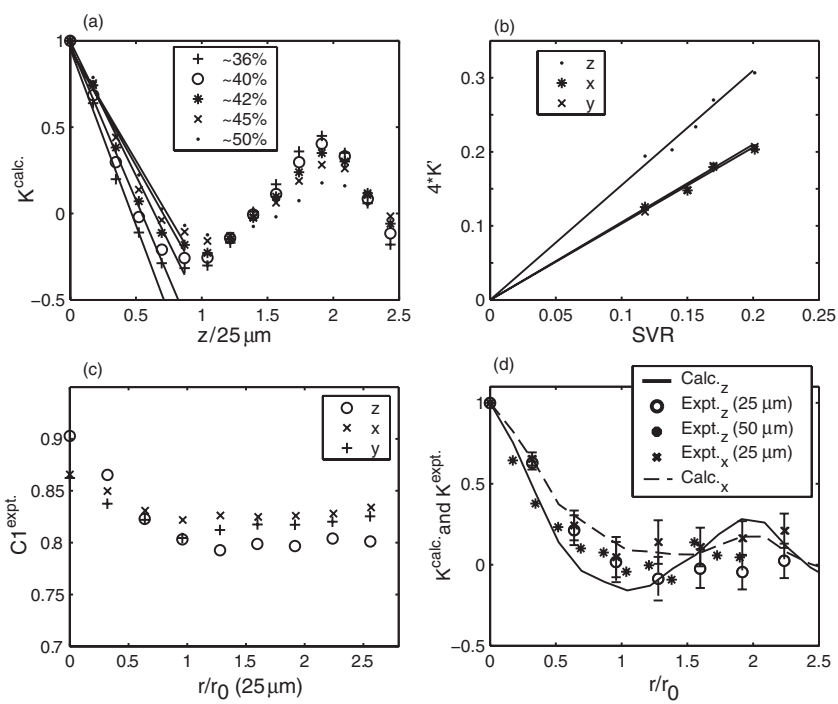

FIG. 4. (a) Numerically calculated internal field correlation $K^{\text {calc }}$ with different porosities. (b) Plot of the initial slope of $K^{\text {calc }}$ as a function of SVR obtained by varying the porosity of the pack [22] (SVR vs $4 K^{\prime}$ ). (c) Plot of measured $C 1\left(r_{\|}\right)$along different applied gradient directions obtained with $t=500 \mu \mathrm{s}$ and $\Delta=70 \mathrm{~ms}$ for the $25 \mu \mathrm{m}$ beads sample. (d) Comparison of experimental measurements of $K$ and numerical calculations.

ratio is found to be $2.2: 0.92: 1$ along the $z, x$, and $y$ directions in a reasonable agreement with the numerical evaluation (1.8:0.97:1). The small difference between $x$ and $y$ directions is likely due to numerical noise. The anisotropy between $z$ and $x, y$ was not reported before [14]. Figure 4(d) shows a comparison between the numerical and experimentally measured $K$ for different bead sizes and the gradient directions. The anisotropy in the numerical $K$ is not reflected in the experimental measurement possibly due to the substantial error bar of the experimental data. It is also possibly due to the difference between the random packs used in numerical and experimental work.

Surface-to-volume ratios were obtained using the measurement of $K$ and Eq. (10): $0.14 \pm 0.02 \mu \mathrm{m}^{-1}, 0.123 \pm$ $0.017 \mu \mathrm{m}^{-1}$, and $0.077 \pm 0.018 \mu \mathrm{m}^{-1}$ for the $25 \mu \mathrm{m}$ ( $x$ gradient), $25 \mu \mathrm{m}$ ( $z$ gradient), and the $50 \mu \mathrm{m}$ ( $z$ gradient) beads, respectively. Porosity of the two samples was measured by NMR $1 \mathrm{D}$ profiling in order to obtain independently $\quad \mathrm{SVR}=3(1 / \phi-1) / r_{0} \quad$ [22]: $\quad 0.138 \pm$ $0.015 \mu \mathrm{m}^{-1}$ and $0.072 \pm 0.01 \mu \mathrm{m}^{-1}$, in good agreement with those from the correlation measurements.

In comparison to other NMR techniques for porous media structure, such as Refs. $[9,19,23]$, the method reported here directly measures the magnetic structure which is a potentially important complement to the physical structure in understanding system functions of (e.g., biological) materials. Furthermore, internal field is ubiquitous in all porous materials and could be directly used to characterize such materials [16]. This Letter combines experimental and numerical study of the internal field to demonstrate a quantitative relationship between the internal field and the porous structure laying the foundation for further development of methodologies based on internal field.

The authors thank E.E. Sigmund, P. N. Sen, S. Ryu, P. Singer, M. D. Hurlimann, L. Zielinski, and D.L. Johnson for discussions. This work is supported in part by NIH (Grant No. EB003869).

*Current address: Memorial Sloan-Kettering Cancer Center, 1275 York Ave., New York, NY 10021, USA.

[1] H. M. Jaeger, S. R. Nagel, and R. P. Behringer, Rev. Mod. Phys. 68, 1259 (1996).

[2] P. G. de Gennes, Rev. Mod. Phys. 71, S374 (1999).

[3] S. Torquato, T. M. Truskett, and P. G. Debenedetti, Phys. Rev. Lett. 84, 2064 (2000).

[4] M. E. Cates, J. P. Wittmer, J.-P. Bouchard, and P. Claudin, Phys. Rev. Lett. 81, 1841 (1998).

[5] A. J. Liu and S. R. Nagel, Nature (London) 396, 21 (1998).

[6] C. H. Liu and S.R. Nagel, Phys. Rev. Lett. 68, 2301 (1992).

[7] P. B. Corson, J. Appl. Phys. 45, 3159 (1974).

[8] J. G. Berryman and S.C. Blair, J. Appl. Phys. 60, 1930 (1986).

[9] P. P. Mitra, P. N. Sen, L. M. Schwartz, and P. L. Doussal, Phys. Rev. Lett. 68, 3555 (1992).

[10] P. N. Sen and M. D. Hürlimann, J. Chem. Phys. 101, 5423 (1994).

[11] N. Martys and E. J. Garboczi, Phys. Rev. B 46, 6080 (1992).

[12] J. H. Jensen, R. Chandra, A. Ramani, H. Lu, G. Johnson, S. P. Lee, K. Kaczynski, and J. A. Helpern, Magn. Reson. Med. 55, 1350 (2006).

[13] D. L. Johnson, J. Koplik, and L. M. Schwartz, Phys. Rev. Lett. 57, 2564 (1986).

[14] B. Audoly, P. N. Sen, S. Ryu, and Y. Q. Song, J. Magn. Reson. 164, 154 (2003).

[15] J. Kärger and W. Heink, J. Magn. Reson. 51, 1 (1983).

[16] Y.-Q. Song, S. Ryu, and P. N. Sen, Nature (London) 406, 178 (2000).

[17] N. V. Lisitza and Y. Q. Song, Phys. Rev. B 65, 172406 (2002).

[18] P. N. Sen and M. D. Hurlimann, J. Chem. Phys. 101, 5423 (1994).

[19] P. T. Callaghan, A. Coy, D. MacGowan, K. J. Packer, and F. O. Zelaya, Nature (London) 351, 467 (1991).

[20] P. T. Callaghan, D. MacGowan, K. J. Packer, and F. O. Zelaya, J. Magn. Reson. 90, 177 (1990).

[21] J. L. Finney, Ph.D. thesis, University of London, 1964.

[22] L. L. Latour, P. P. Mitra, R. L. Kleinberg, and C. H. Sotak, J. Magn. Reson., Ser. A 101, 342 (1993).

[23] G. A. Barrall, L. Frydman, and G. C. Chingas, Science 255, 714 (1992). 